\title{
MicroRNA-214 targets Wnt3a to suppress liver cancer cell proliferation
}

\author{
YANG YANG $^{1 *}$, ZHENGHAO ZHAO $^{2 *}$, NI HOU ${ }^{2},{\text { YULONG } \mathrm{LI}^{3}, \text { XIAOFEI WANG }}^{2}$, FEI WU $^{2}$, \\ RUIFANG SUN ${ }^{4}$, JIA HAN ${ }^{2}$, HONGFEI SUN ${ }^{2}$, TUSHENG SONG ${ }^{2}$, CHEN HUANG $^{2}$ and YUAN SHAO 5 \\ ${ }^{1}$ Department of Health Toxicology and Hygiene Inspection, School of Public Health, Xi'an Jiaotong University \\ Health Science Center; ${ }^{2}$ Department of Cell Biology and Genetics, School of Basic Medical Sciences, \\ Xi'an Jiaotong University Health Science Center, Xi'an Jiaotong University, Xi'an, Shaanxi 710061; \\ ${ }^{3}$ Department of Gastroenterology, Shaanxi Provincial People's Hospital, Xi'an, Shaanxi 710068; \\ ${ }^{4}$ Department of Pathology, School of Basic Medical Sciences, Xi'an Jiaotong University Health Science Center, \\ Xi'an Jiaotong University; ${ }^{5}$ Department of Otorhinolaryngology, First Affiliated Hospital \\ of Xi'an Jiaotong University, Xi'an, Shaanxi 710061, P.R. China
}

Received October 24, 2016; Accepted June 20, 2017

DOI: $10.3892 / \mathrm{mmr} .2017 .7483$

\begin{abstract}
MicroRNAs (miRNAs/miRs) are crucial molecules that act as tumor suppressor genes or oncogenes in human cancer progression. The dysregulation of miRNA expression has been detected in liver cancer. The present study aimed to explore the molecular mechanisms by which miR-214 affects liver cancer cell proliferation. Reverse transcription-quantitative polymerase chain reaction was used to determine the expression of miR-214 in liver cancer cell lines and hepatocellular carcinoma (HCC) tissues. A luciferase reporter assay was performed to determine whether Wnt3a is a target gene of miR-214. Cell Counting kit-8 and cell cycle analysis were used to explore the effects of miR-214 on liver cancer cell proliferation. Immunohistochemistry was used to detect protein expression levels. Wnt3a knockdown was used to determine the function of Wnt3a in liver cancer cell proliferation. The results demonstrated that the expression levels of human miR-214 were reduced in HCC tissues and liver cancer cell lines compared with in control tissues and cells. Overexpression of miR-214 and Wnt3a silencing each
\end{abstract}

Correspondence to: Professor Chen Huang, Department of Cell Biology and Genetics, School of Basic Medical Sciences, Xi'an Jiaotong University Health Science Center, Xi'an Jiaotong University, 76 Yanta Western Road, Xi'an, Shaanxi 710061, P.R. China

E-mail: hchen@mail.xjtu.edu.cn

Dr Yuan Shao, Department of Otorhinolaryngology, First Affiliated Hospital of Xi'an Jiaotong University, 277 Yanta Western Road, Xi'an, Shaanxi 710061, P.R. China

E-mail: shaxiaoying1976@163.com

${ }^{*}$ Contributed equally

Key words: microRNA-214, hepatocellular carcinoma, proliferation, Wnt3a inhibited liver cancer cell growth. Conversely, inhibition of miR-214 promoted liver cancer cell growth. The present study indicated that miR-214 acts as a tumor suppressor and may be considered a promising therapeutic target for the treatment of liver cancer.

\section{Introduction}

Hepatocellular carcinoma (HCC) is one of the most common types of malignancy worldwide, with $\sim 630,000$ new cases reported each year (1). HCC development is a complex process that is associated with numerous risk factors, including several known environmental factors, hepatitis, and alcohol and tobacco consumption $(2,3)$. There is increasing evidence supporting the role of genetic factors in HCC risk (4). The lack of novel therapeutic strategies for the treatment of HCC emphasizes the need to determine the molecular mechanisms underlying liver cancer development. MicroRNAs (miRNAs/miRs) are a class of endogenous, non-coding RNAs 18-22 nucleotides in length, which are crucial for gene expression regulation (5). In the present study, differential miRNA expression between tumor tissues and normal tissues was identified to be associated with cancer progression via target gene regulation. More than half of miRNAs are located in tumor-associated genomic regions or fragile sites (6), and miRNAs may be classified as tumor suppressors or oncogenes based on their modulation of oncogenic and tumor suppressor pathways (7). Numerous miRNAs are involved in liver cancer development, proliferation, apoptosis and differentiation (8). For example, miR-150-5p inhibits hepatoma cell migration and invasion by targeting matrix metalloproteinase 14 (9) and miR-486-5p suppresses tumor growth in $\mathrm{HCC}$ by targeting phosphatidylinositol 3-kinase regulatory subunit $\alpha$ (10).

The present study demonstrated that miR-214 expression is lower in HCC tissues and liver cancer cell lines compared with in matched normal tissues and the HL-7702 cell line, 
indicating its role as a tumor suppressor in liver cancer. In addition, Wnt3a was confirmed as a target gene of miR-214. Using immunohistochemistry, the present study indicated that the expression of Wnt3a was higher in HCC tissues compared with in normal tissues. In addition, the function of miR-214 in liver cancer cell proliferation was investigated, and overexpression of miR-214 and Wnt3a silencing arrested the liver cancer cell cycle at $G_{1}$ phase. These results demonstrated that miR-214 may suppress the growth of liver cancer cells by targeting Wnt3a.

\section{Materials and methods}

Human tissue samples and cell lines. The SMMC-7721, HepG2 and Hep3B liver cancer cell lines, and the HL-7702 normal liver cell line were provided by the Key University, Ministry of Education (Xi'an, China). These cells were cultured in Dulbecco's modified Eagle's medium (Thermo Fisher Scientific, Inc., Waltham, MA, USA) supplemented with $10 \%$ fetal bovine serum (Thermo Fisher Scientific, Inc.) at $37^{\circ} \mathrm{C}$ in a humidified chamber containing $5 \% \mathrm{CO}_{2}$. Tumor tissues and matched normal tissues were obtained from 24 HCC patients (19 males, 5 females; age, 21-72 years). Patients were enrolled between September 2011 and January 2013 at the First Affiliated Hospital of Xi'an Jiaotong University (Xi'an, China). All patients provided written informed consent. The present study was approved by the Medical Ethical Committee of the College of Medicine, Xi'an Jiaotong University (Xi'an, China).

$R N A$ extraction and reverse transcription-quantitative polymerase chain reaction $(R T-q P C R)$. Total RNA was extracted using TRIzol (Invitrogen; Thermo Fisher Scientific, Inc.) from the cells or tissues according to the manufacturer's protocol. cDNA synthesis was performed using the Prime-Script RT reagent kit (Takara Biotechnology Co., Ltd., Dalian, China) according to the manufacturer's protocol. qPCR was performed on cDNA using SYBR Premix Ex Taq (Takara Biotechnology Co., Ltd.) according to the manufacturer's protocol. PCR amplification was performed on a FTC-3000TM system (Funglyn Biotech Inc., Toronto, ON, Canada). Thermocycling conditions were as follows: Initial denaturation at $95^{\circ} \mathrm{C}$ for $30 \mathrm{sec}$, followed by 40 cycles at $95^{\circ} \mathrm{C}$ for $5 \mathrm{sec}$ and at $60^{\circ} \mathrm{C}$ for $30 \mathrm{sec}$. U6 was used for normalization in miRNA detection. The $2^{-\Delta \Delta C q}$ method was used to quantify the relative expression levels of miR-214 (11). The primer sequences are listed in Table I.

Plasmid vector constructs. EcoRI and HindIII sites were inserted into the multiple cloning sites of the pcDNA6.2-GW/EmGFP vector (Invitrogen; Thermo Fisher Scientific, Inc.). The primary miR-214 sequence was amplified by PCR from genomic DNA, as aforementioned, and then cloned into the EcoRI and HindIII sites of the pcDNA6.2-GW/EmGFP vector. The wild-type (wt) and mutant (mut) Wnt3a 3' untranslated region (UTR) sequences with SacI and $X h o I$ restriction enzymes were synthesized by Sangon Biotech. Co., Ltd. (Shanghai, China) and cloned between the SacI and XhoI sites of pmirGLO Dual-Luciferase miRNA Target Expression Vector (Promega Corporation, Madison, WI, USA). The 3'UTR sequences are listed in Table I. Wnt3a
Table I. Sequences of primers, inhibitors and UTRs used in the present study.

Name $\quad$ Sequence (5'-3')

U6

RT: CGCTTCACGAATTTGCGTGTCAT Forward: GCTTCGGCAGCACATATACTA AAAT

Reverse: CGCTTCACGAATTTGCGTGT CAT

$\operatorname{miR}-214$

RT: GTCGTATCCAGTGCGTGTCGTGG AGTCGGCAATTGCACTGGATACGACA CTGCCT

Forward: ATCCAGTGCGTGTCGTG

Reverse: TGCTACAGCAGGCACAGAC

miR-214ACTGCCTGTCTGTGCCTGCTGT

inhibitor

miR-214-

CAGTACTTTTGTGTAGTACAA

inhibitor-ctrl

si-ctrl-S

UUCUCCGAACGUGUCACGUTT

si-ctrl-AS

siWnt3a-S

siWnt3a-AS

ACGUGACACGUUCGGAGAATT

Wnt3a

CCCACUCGGAUACUUCUUATT

3'UTR-WS

Wnt3a

3'UTR-WA

UAAGAAGUAUCCGAGUGGGTT

CCCTGCCAGGGAACTGGCCTGCTGCC

Wnt3a

3'UTR-MS

Wnt3a

3'UTR-MA

TCGAGGCAGCAGGCCAGTTCCCTGG CAGGGAGCT

CCCTGCCAGGGAACTGGCCACGTGCC

RT, reverse transcriptase; miR, microRNA; UTR, untranslated region; S, sense; AS, antisense; WS, wild-type sense strand; WA, wild-type antisense strand; MS, mutated sense strand; MA, mutated antisense strand.

was cloned into a GV230 vector (Shanghai GeneChem Co., Ltd., Shanghai, China).

Luciferase activity assay. Potential target genes for miR-214 were predicted using RegRNA online software version 2.0 (http://regrna2.mbc.nctu.edu.tw). A potential miR-214-binding site in the 3'-UTR of Wnt3a mRNA was identified. To determine whether Wnt3a was a direct target of miR-214, HepG2 cells were co-transfected with miR-214 and wt Wnt3a 3'UTR or mut Wnt3a 3'UTR pmirGLO plasmid, using Lipofectamine 2000 (Invitrogen; Thermo Fisher Scientific, Inc.) as the transfection reagent, according to manufacturer's protocol. HepG2 cells co-transfected with miR-214 and pmirGLO vector were used as the control. After $48 \mathrm{~h}$, luciferase activity was detected using the Dual-Luciferase Reporter Assay system (Promega Corporation) according to the manufacturer's protocol.

Cell proliferation assay. Cells were seeded into 96-well plates at a density of 5,000 cells/well. Cells were transfected with miR-214, miR-214 inhibitor, small interfering RNA(si) 

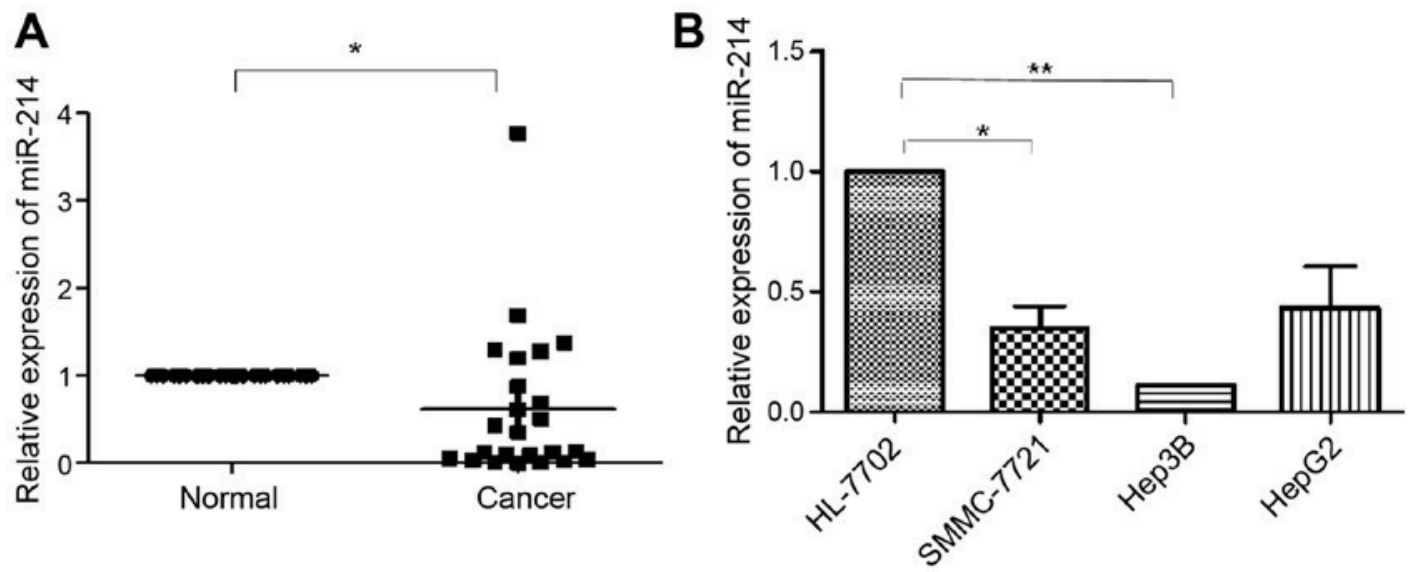

C

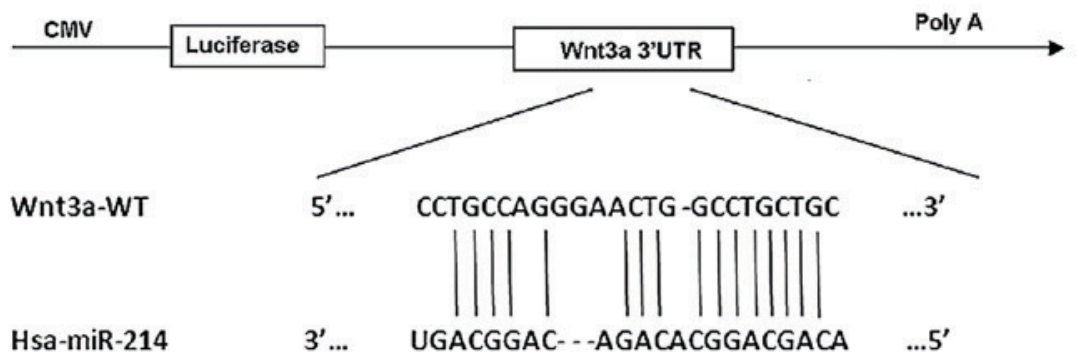

Wnt3a-MT $\quad 5^{\prime} \ldots \quad$ CCTGCCAGGGAACTG-GCCACGTGC $\quad \ldots 3^{\prime}$

D

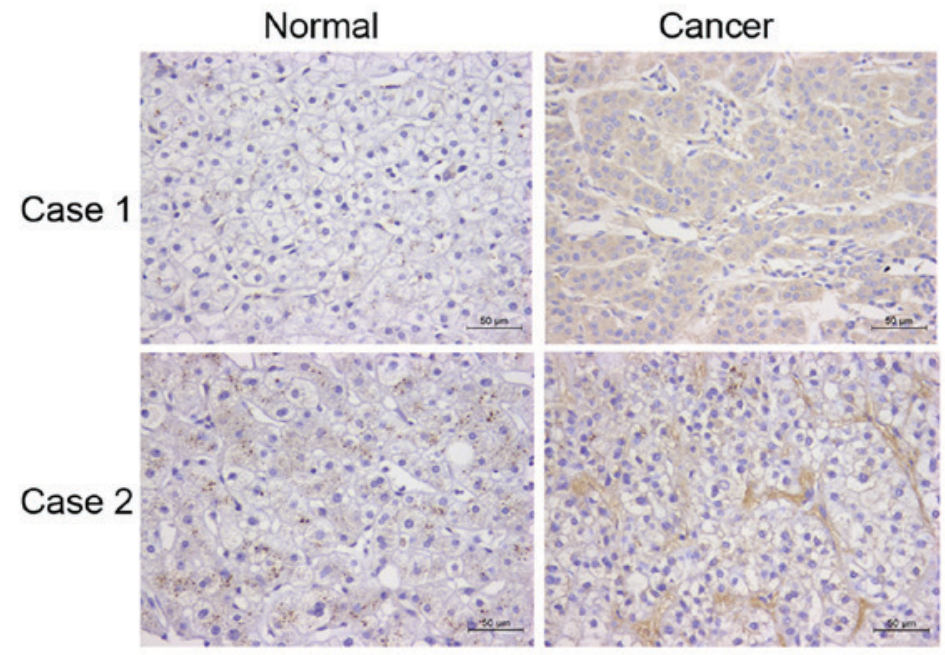

E
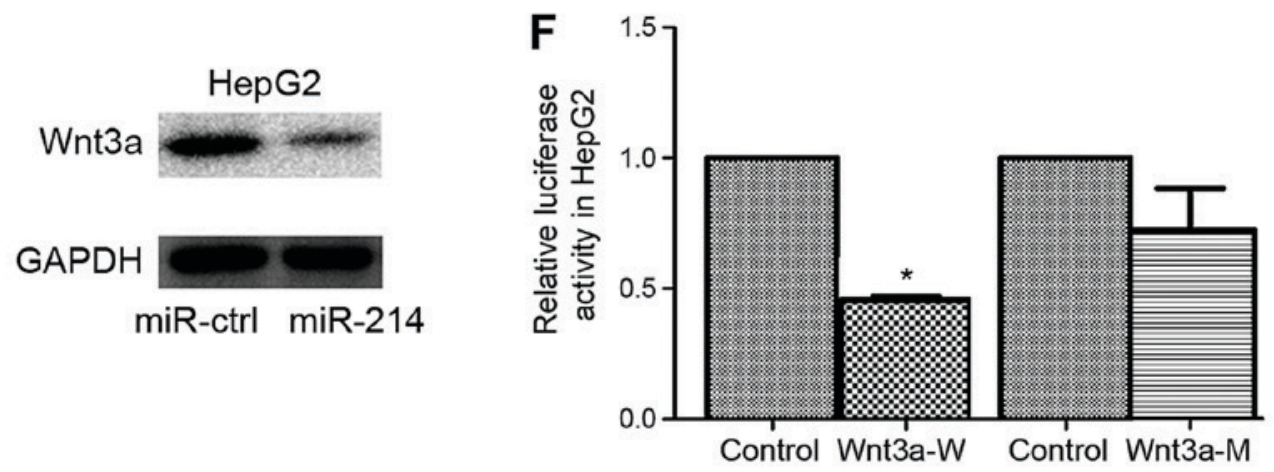

Figure 1. miR-214 is downregulated in liver cancer and targets Wnt3a. (A) Reverse transcription-quantitative polymerase chain reaction was performed to examine the expression of miR-214 in 24 paired human hepatocellular carcinoma and non-tumor tissues. (B) Relative expression of miR-214 in liver cancer cell lines and a normal liver cell line. ${ }^{* *} \mathrm{P}<0.01$; ${ }^{*} \mathrm{P}<0.05$. (C) miR-214 seed region sequence in the $3^{\prime} \mathrm{UTR}$ of Wnt3a. (D) Wnt3a protein expression as detected by immunohistochemistry. (E) Protein expression levels of Wnt3a were measured by western blot analysis in HepG2 cells transfected with miR-214 or miR-ctrl. (F) miR-214 was co-transfected with pmirGLO, pmirGLO-Wnt3a-3'-UTR-wt or pmirGLO-Wnt3a-3'-UTR-mut in HepG2 cells. Relative luciferase activity was measured after 48 h. "P<0.05 vs. control. miR, microRNA; mut/M, mutant; UTR, untranslated region; wt/W, wild-type. 
A

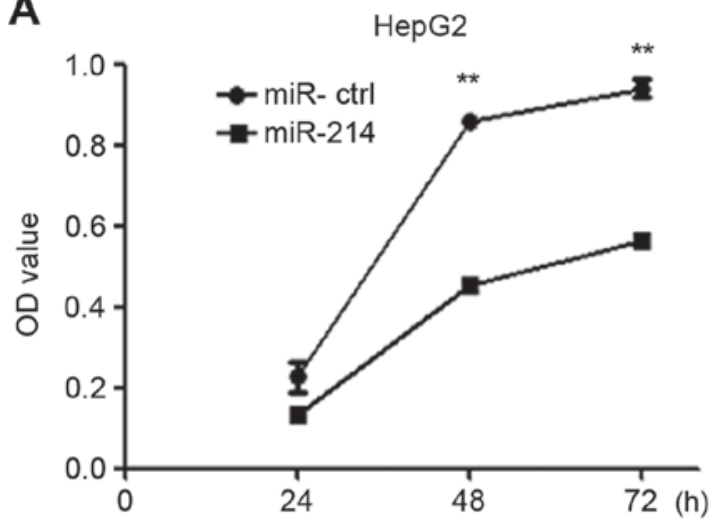

C

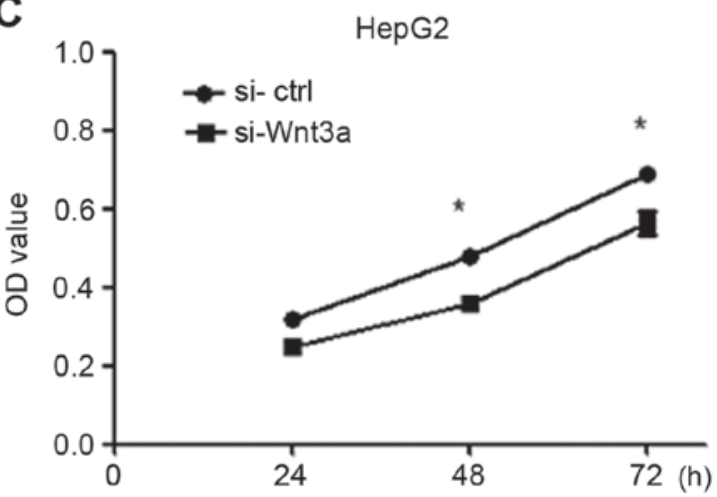

E

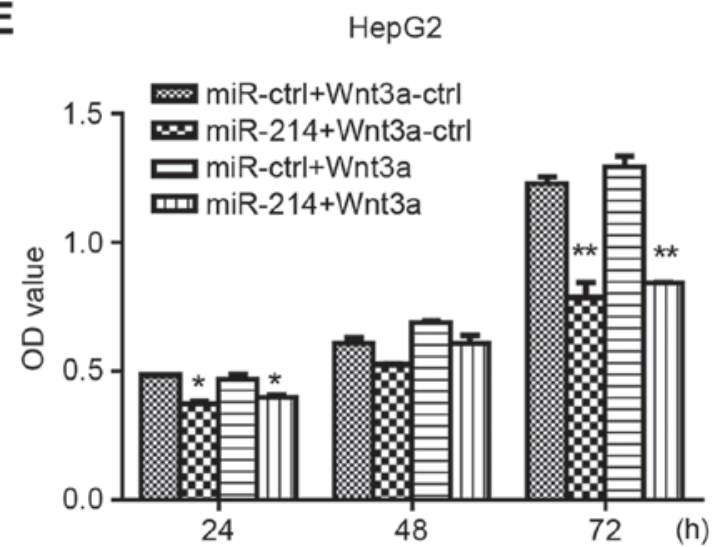

B Нер3B

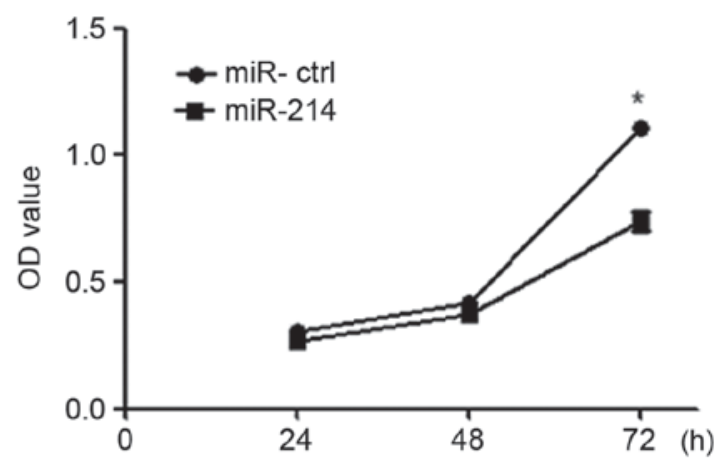

D

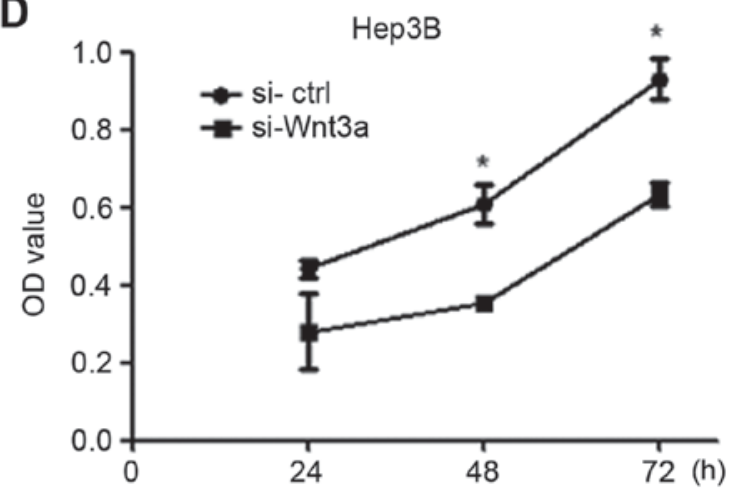

$\mathbf{F}$ Нер3В

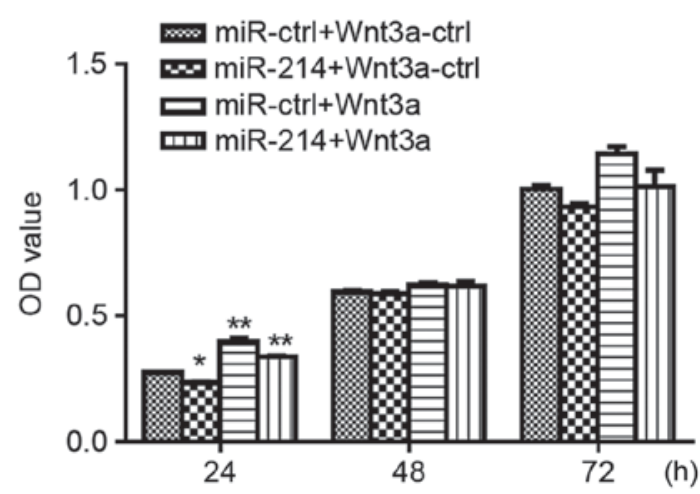

Figure 2. miR-214 inhibits the proliferation of liver cancer cells. CCK8 assay was performed to detect the effects of miR-214 on cell proliferation at 24,48 , and $72 \mathrm{~h}$ in (A) HepG2 and (B) Hep3B cells. CCK8 assay was performed to detect the effects of siWnt3a on cell proliferation at 24, 48 and $72 \mathrm{~h}$ in (C) HepG2 and (D) Hep3B cells. Wnt3a overexpression vector was co-transfected with miR-ctrl or miR-214 into (E) HepG2 and (F) Hep3B cells, and cell proliferation was detected by CCK8 assay. ${ }^{*} \mathrm{P}<0.05 ;{ }^{* *} \mathrm{P}<0.01$ vs. miR-ctrl + Wnt3a-ctrl. CCK8, Cell Counting kit-8; ctrl, control; miR, microRNA; OD, optical density; si, small interfering RNA.

Wnt3a, or their respective controls using Lipofectamine 2000 as the transfection reagent, according to manufacturer's protocol. The miR-214 inhibitor was synthesized by Sangon Biotech Co., Ltd. The siWnt3a and the corresponding control (ctrl) siRNA were purchased from Shanghai GenePharma Co., Ltd. (Shanghai, China). Cell Counting kit-8 (CCK8; 7Sea Biotech, Shanghai, China) assay was performed according to the manufacturer's protocol. Optical density at $450 \mathrm{~nm}$ was measured 24,48 and $72 \mathrm{~h}$ post-transfection using the FLUOstar OPTIMA microplate reader (BMG Labtech $\mathrm{GmbH}$, Ortenberg, Germany), in order to measure cell proliferation.
Cell cycle analysis. HepG2 and Hep3B cells were transfected with miR-214, miR-control (ctrl), miR-214 inhibitor, inhibitor-ctrl, siWnt3a or si-ctrl (Shanghai GenePharma Co., Ltd.) using Lipofectamine 2000 according to the manufacturer's instructions. At $48 \mathrm{~h}$ post-transfection, $1 \times 10^{6}$ cells were harvested and washed in PBS, then fixed with $70 \%$ ice-cold ethanol at $4^{\circ} \mathrm{C}$ overnight. The cells were washed in PBS and then incubated at $4^{\circ} \mathrm{C}$ with $0.1 \mathrm{mg} / \mathrm{ml} \mathrm{RNase} \mathrm{A} \mathrm{and} 0.05 \mathrm{mg} / \mathrm{ml}$ propidiumiodide(PI; Sigma-Aldrich;MerckKGaA,Darmstadt, Germany) for $30 \mathrm{~min}$. Fluorescence-activated cell sorting was performed using a BD FACSort flow cytometer (BD 
Biosciences, Franklin Lakes, NJ, USA). ModFit LT version 4.0 (Verity Software House, Inc., Topsham, ME, USA) was used to analyze cell cycle distributions.

Immunohistochemistry. Formaldehyde-fixed, paraffinembedded tissue samples were sectioned at $5 \mu \mathrm{m}$, after which the samples were deparaffinized in xylene and hydrated using graded alcohol. Antigen retrieval was performed with citrate buffer $0.01 \mathrm{M}$ (pH 6.0), followed by blocking endogenous peroxidases with $3 \% \mathrm{H}_{2} \mathrm{O}_{2}$ for $20 \mathrm{~min}$, and blocking of non-specific binding with normal goat serum (Beijing Biosynthesis Biotechnology Co., Ltd., Beijing, China) at room temperature for $20 \mathrm{~min}$. The slides were then incubated with anti-Wnt3a primary antibodies [FT20][a21] (1:100; Beijing Biosynthesis Biotechnology Co., Ltd., Beijing, China, bs-1700R) at $4^{\circ} \mathrm{C}$ overnight, followed by incubation with the HRP-conjugated secondary antibody (Beijing ZhongShan-Golden Bridge Biological Technology Co., Ltd., Beijing, China) [FT22][a23] for $30 \mathrm{~min}$ at $37^{\circ} \mathrm{C}$. Detection was performed using 3,3'-diaminobenzidine [FT25][a26] for $3 \mathrm{~min}$ at room temperature (Beijing ZhongShan-Golden Bridge Biological Technology Co., Ltd., Beijing, China) and Harris hematoxylin [FT27][a28] for $30 \mathrm{sec}$ at room temperature. Finally, a Leica Photo Microscope (Leica Microsystems $\mathrm{GmbH}$, Wetzlar, Germany) was used to obtain digital images.

Western blot analysis. Total proteins were extracted using radioimmunoprecipitation assay lysis buffer (Xi'an Wolsen Biotechnology Co., Ltd., Xi'an, China). Protein concentration was determined using a NanoDrop ND-1000 spectrophotometer (NanoDrop; Thermo Fisher Scientific, Inc., Wilmington, DE, USA). Equal amounts ( $30 \mu \mathrm{g})$ of extracted protein samples were separated by $10 \%$ SDS-PAGE and were then transferred onto a polyvinylidene fluoride membrane, which was blocked with $5 \%$ non-fat milk in TBS containing $0.05 \%$ Tween-20 (TBST) for $1 \mathrm{~h}$ at room temperature. Membranes were then incubated with rabbit anti-human Wnt3a (cat no. bs-1700R; 1:100; Beijing Biosynthesis Biotechnology Co., Ltd.) and rabbit anti-human GAPDH (cat no. 10494-1-AP; 1:2,000; ProteinTech Group, Inc., Chicago, IL, USA) at $4^{\circ} \mathrm{C}$ overnight. Subsequently, the membrane was washed 3 times with TBST and incubated with secondary goat anti-rabbit antibody (cat no. 111-035-144; 1:1,000; Jackson ImmunoResearch Laboratories, Inc., West Grove, PA, USA) for $2 \mathrm{~h}$ at room temperature. Protein bands were visualized using Immobilon Western Chemiluminescent HRP substrate (EMD Millipore, Billerica, MA, USA). Blots were semi-quantified by densitometry using Quantity One imaging software (Bio-Rad Laboratories, Inc., Hercules, CA, USA).

Statistical analysis. Statistical analysis was performed with SPSS software version 13.0 (SPSS, Inc., Chicago, IL, USA). Student's t-test or one-way analysis of variance followed by a post hoc Tukey test were used to analyze the data from three independent experiments. $\mathrm{P}<0.05$ was considered to indicate a statistically significant difference.

\section{Results}

miR-214 is downregulated in liver cancer and targets Wnt3a. To explore the expression of miR-214 in liver cancer development, the expression levels of miR-214 were detected in 24 pairs of $\mathrm{HCC}$ and matched normal tissue samples using RT-qPCR. miR-214 was significantly downregulated in HCC tissue samples compared with in matched non-tumor tissues, as presented in Fig. 1A. In addition, miR-214 was downregulated in the examined liver cancer cells (SMMC-7721, Hep3B, HepG2) compared with in normal HL-7702 hepatocytes (Fig. 1B). These findings suggested that miR-214 was downregulated in HCC tissues and liver cancer cell lines; therefore, miR-214 may act as a potential anti-oncogenic miRNA in liver cancer.

The prediction of miR-214 targets was acquired using RegRNA. Wnt3a was identified as a potential target gene of miR-214. A miR-214 binding site was identified in the 3'UTR of Wnt3a (Fig. 1C). Immunohistochemistry demonstrated that Wnt3a protein expression was increased in HCC samples compared with in normal tissues (Fig. 1D). The protein expression levels of Wnt3a were also measured by western blotting in HepG2 cells transfected with miR-214 or miR-ctrl, and the results demonstrated that miR-214 overexpression was able to reduce Wnt3a protein expression (Fig. 1E). Dual-luciferase reporter assay was employed to determine whether Wnt3a was a direct target of miR-214. Luciferase activity was significantly reduced following co-transfection with miR-214 and wt Wnt3a 3'UTR compared with the control; however, luciferase activity was not altered following co-transfection with miR-214 and mut Wnt3a 3'UTR (Fig. 1F). These results provided direct evidence that Wnt3a was a target of miR-214.

miR-214 suppresses liver cancer cell growth via Wnt3a targeting. To explore the role of miR-214 in liver cancer cell growth, HepG2 and Hep3B cells were transfected with miR-214, miR-ctrl, siWnt3a or si-ctrl. Overexpression of miR-214 suppressed the growth of HepG2 and Hep3B cells following transfection for 24,48 and $72 \mathrm{~h}$ compared with control vector-transfected cells (Fig. 2A and B). The results of Wnt3a silencing were consistent with those of miR-214 overexpression. Based on CCK8 assays (Fig. 2C and D), siWnt3A inhibited the growth of HepG2 and Hep3B cells. To further demonstrate that miR-214 suppressed the growth of HepG2 and Hep3B by targeting Wnt3a, Wnt3a overexpression vector and miR-ctrl or miR-214 were co-transfected into HepG2 and Hep3B cells. The results demonstrated that overexpression of Wnt3a can mitigate the growth inhibition caused by overexpression of miR-214 (Fig. 2E and F).

Overexpression of miR-214 and silencing of Wnt3a inhibits liver cancer cell proliferation via cell cycle regulation. HepG2 and Hep3B cells were transfected with miR-214 or miR-ctrl, and siWnt3a or si-ctrl. Cell cycle distribution, as detected by flow cytometry, revealed an accumulation of HepG2 and Hep3B cells in $\mathrm{G}_{1}$ phase in the miR-214 overexpression groups compared with the miR-ctrl groups (Fig. 3A and B). In addition, the number of cells in $G_{1}$ phase was increased following transfection with siWnt3a compared with the control groups (Fig. 3C and D). These results suggested that overexpression of miR-214 or Wnt3a silencing may induce $\mathrm{G}_{1}$ cell cycle arrest.

Knockdown of miR-214 contributes to liver cancer cell proliferation. miR-214 inhibitor was used to further investigate the 

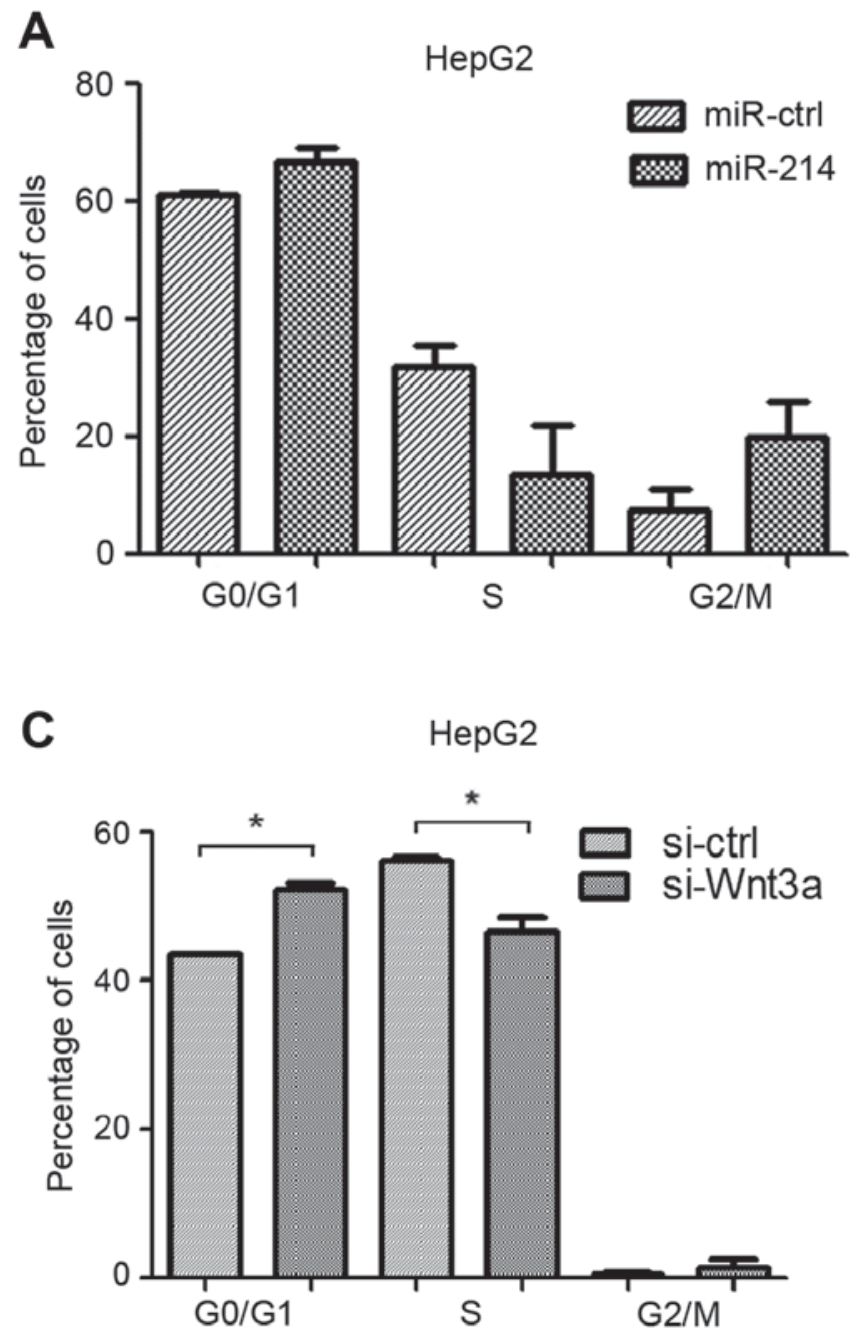

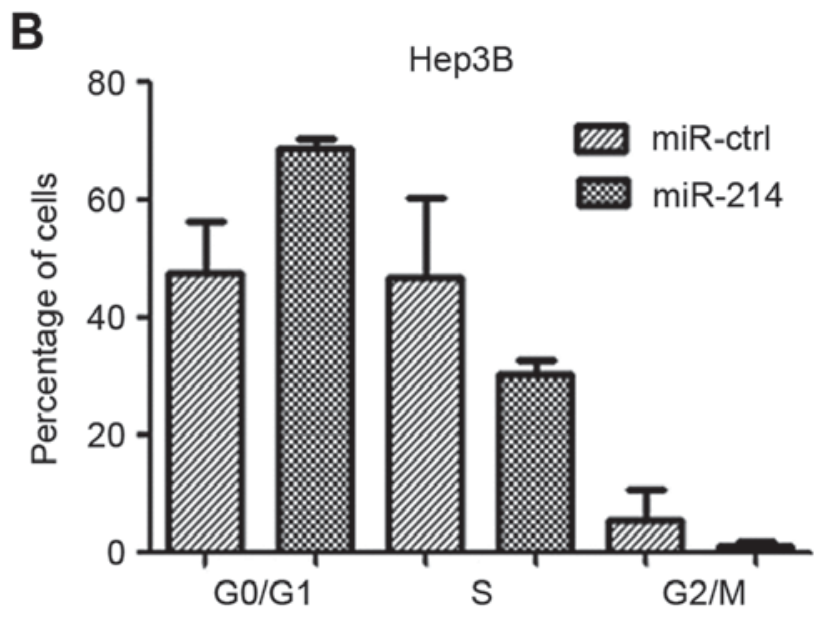

D

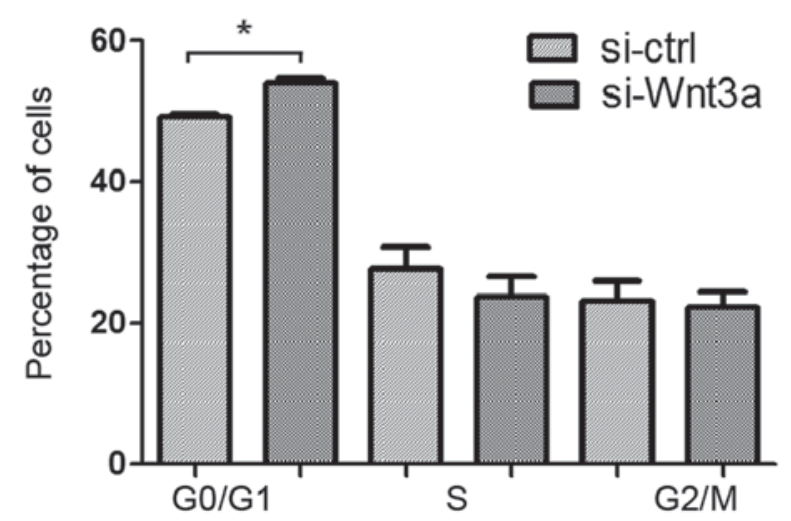

Figure 3. Overexpression of miR-214 or Wnt3a silencing affects cell cycle progression. Cell cycle analysis of (A) HepG2 and (B) Hep3B cells following transfection with miR-214 or miR-ctrl for 48 h. Cell cycle analysis of (C) HepG2 and (D) Hep3B cells following transfection with siWnt3a or si-ctrl for 48 h. ${ }^{*} \mathrm{P}<0.05$. ctrl, control; miR, microRNA; si, small interfering RNA.

effects of miR-214 silencing on liver cancer cell proliferation. HepG2 and Hep3B cells were transfected with miR-214 inhibitor or inhibitor-ctrl, and the expression levels of miR-214 were detected (Fig. 4A and B). Cell viability was measured using the CCK8 assay; transfection with the miR-214 inhibitor increased the proliferation of HepG2 cells compared with the control group (Fig. 4C and D). The number of cells in G1 phase was reduced in the Hep3B cells transfected with miR-214 inhibitor compared with the control group (Fig. 4E and F).

\section{Discussion}

Previous studies have supported the role of miRNAs in tumorigenesis, including human liver cancer, and their functions as tumor suppressors or oncogenes $(12,13)$. These small non-protein-coding RNAs are able to target numerous genes, including oncogenes, leading to the degradation of target mRNAs and inhibition of translation (14). miRNA dysregulation is associated with human cancer; specifically, miR-214 is a tumor suppressor of HCC (15). The present study revealed that miR-214 is downregulated in HCC cancer tissues and liver cancer cell lines, and may act as a tumor suppresser via Wnt3a signaling.
The Wnt/ $\beta$-catenin signaling pathway is highly conserved and serves an essential role in regulating a series of genes associated with processes including embryonic development, stem cell maintenance and tissue homeostasis, and its disruption is a common cause of numerous types of cancer (16-18). In addition, the pathway is important during the development of HCC (19). Previous studies have demonstrated that $\beta$-catenin-activating mutations may associate with HCC, thus establishing an association between Wnt signaling and $\mathrm{HCC}(20,21)$ and providing novel insight into the complex network underlying HCC. Wnts act through distinct canonical and noncanonical pathways (22). The canonical Wnt/ $\beta$-catenin signaling pathway is involved in the transition from cell proliferation to myogenic differentiation (23). $\beta$-catenin is a central regulator in the $W n t / \beta$-catenin pathway $(24,25)$ and is present in the plasma membrane, cytoplasm and nucleus. The interplay between the cell cycle and Wnt signaling is complex and requires further study $(26,27)$. Wnt3a is a member of the Wnt family located on human chromosome 17 (17q21) and is an important molecule in the $\mathrm{Wnt} / \beta$-catenin pathway that can active the canonical Wnt signaling pathway (28). The 
A

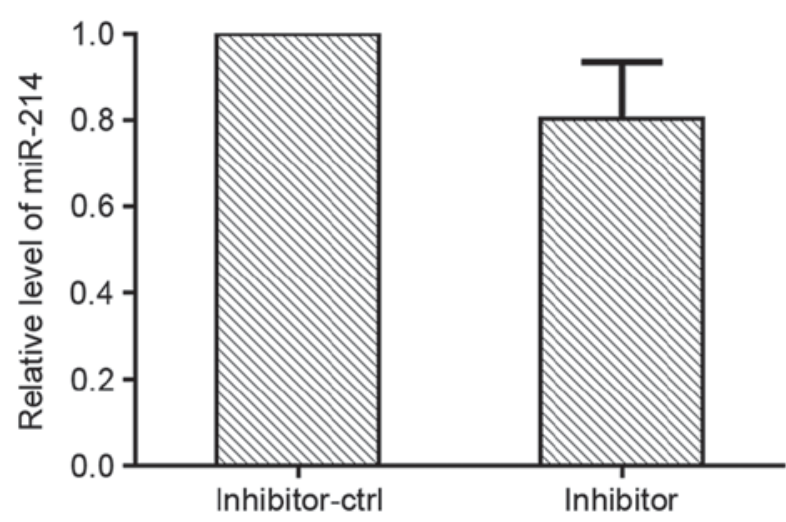

C

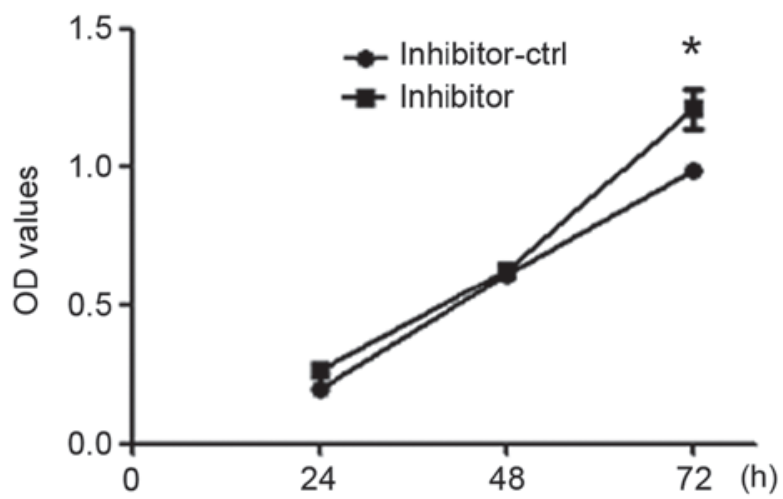

B

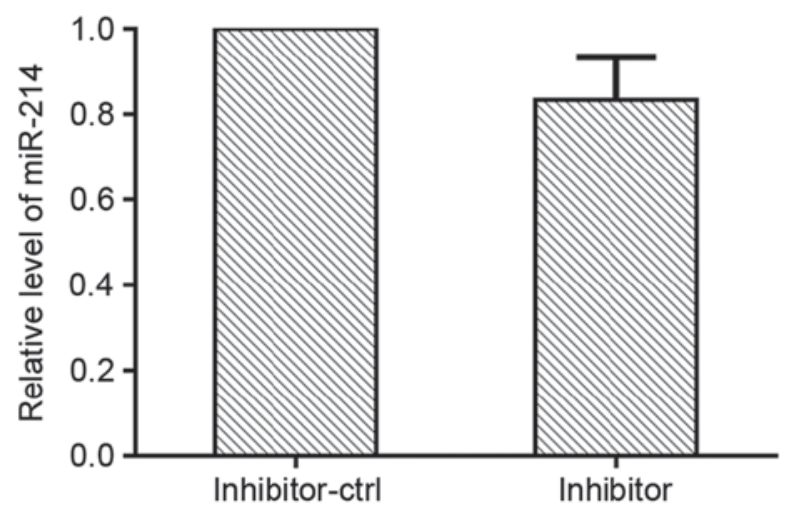

D

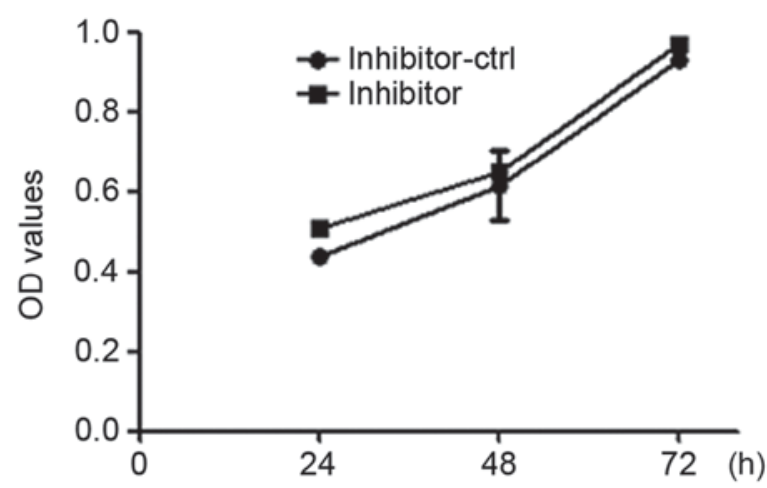

E

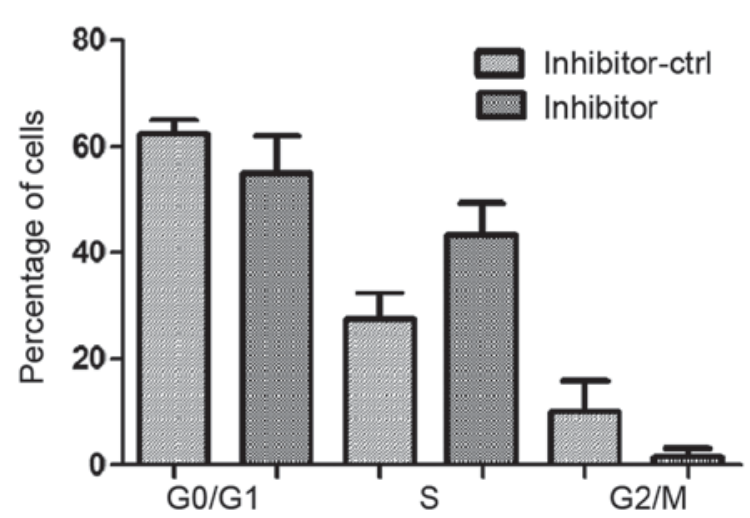

F

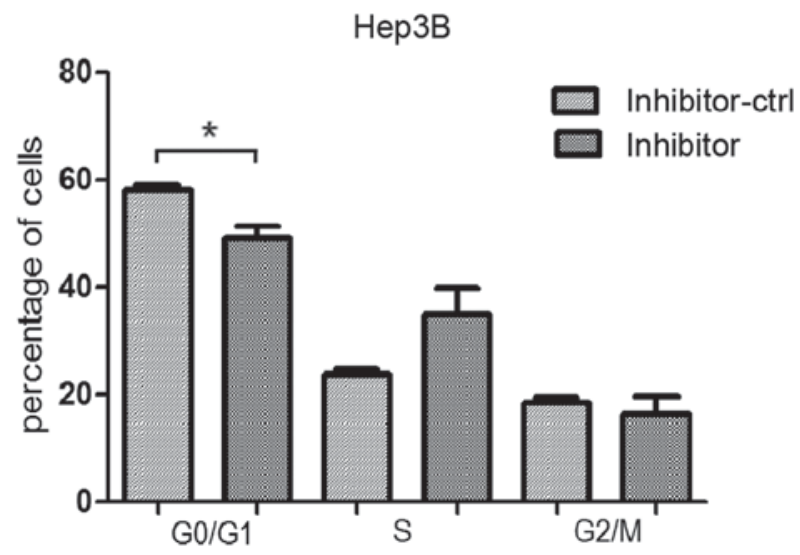

Figure 4. miR-214 inhibitor promotes proliferation of HepG2 cells. (A) HepG2 and (B) Hep3B cells were transfected with inhibitor-ctrl or miR-214 inhibitor for $48 \mathrm{~h}$ and the expression of miR-214 was detected using reverse transcription-quantitative polymerase chain reaction. CCK8 assay was performed to measure the proliferation of (C) HepG2 and (D) Hep3B cells following transfection with miR-214 inhibitor or inhibitor-ctrl. Cell cycle progression of (E) HepG2 and (F) Hep3B cells was determined 48 h post-transfection. ${ }^{*} \mathrm{P}<0.05$. CCK8, Cell Counting kit-8; ctrl, control; miR, microRNA; OD, optical density.

overexpression of Wnt3a expression serves an important role in hepatocarcinogenesis (29).

In conclusion, the present study demonstrated that Wnt3a is a target gene of miR-214. In addition, the results indicated that miR-214 may inhibit cell proliferation in liver cancer cells via Wnt3a targeting. These data provide experimental evidence to suggest that miR-214 acts as a tumor suppressor by suppressing the Wnt/ $\beta$-catenin signaling pathway. Therefore,
miR-214 may be considered a potential molecular therapeutic target for the treatment of liver cancer.

\section{Acknowledgements}

The present study was supported by the Scientific Research and Sharing Platform Construction Project of Shaanxi Province (grant no. 2015FWPT-14) and Research Support 
Project of New Teacher of Xi'an Jiaotong University (grant no. YX1K078).

\section{References}

1. Kew MC: Epidemiology of chronic hepatitis B virus infection, hepatocellular carcinoma, and hepatitis B virus-induced hepatocellular carcinoma. Pathol Biol (Paris) 58: 273-277, 2010.

2. Sherman M: Hepatocellular carcinoma: New and emerging risks. Dig Liver Dis 42 (Suppl 3): S215-S222, 2010.

3. Dragani TA: Risk of HCC: Genetic heterogeneity and complex genetics. J Hepatol 52: 252-257, 2010.

4. Feo F, De Miglio MR, Simile MM, Muroni MR, Calvisi DF, Frau M and Pascale RM: Hepatocellular carcinoma as a complex polygenic disease. Interpretive analysis of recent developments on genetic predisposition. Biochim Biophys Acta 1765: 126-147, 2006.

5. Rajendiran S, Parwani AV, Hare RJ, Dasgupta S, Roby RK and Vishwanatha JK: MicroRNA-940 suppresses prostate cancer migration and invasion by regulating MIEN1. Mol Cancer 13: 250, 2014.

6. Calin GA, Liu CG, Sevignani C, Ferracin M, Felli N, Dumitru CD, Shimizu M, Cimmino A, Zupo S, Dono M, et al: MicroRNA profiling reveals distinct signatures in B cell chronic lymphocytic leukemias. Proc Natl Acad Sci USA 101: 11755-11760, 2004.

7. Ventura A and Jacks T: MicroRNAs and cancer: Short RNAs go a long way. Cell 136: 586-591, 2009.

8. Wiemer EA: The role of microRNAs in cancer: No small matter. Eur J Cancer 43: 1529-1544, 2007.

9. Li T, Xie J, Shen C, Cheng D, Shi Y, Wu Z, Zhan Q, Deng X, Chen H, Shen B, et al: miR-150-5p Inhibits Hepatoma cell migration and invasion by targeting MMP14. PLoS One 9: e115577, 2014.

10. Huang XP, Hou J, Shen XY, Huang CY, Zhang XH, Xie YA and Luo XL: MicroRNA-486-5p, which is downregulated in hepatocellular carcinoma, suppresses tumor growth by targeting PIK3R1. FEBS J 282: 579-594, 2015.

11. Livak KJ and Schmittgen TD: Analysis of relative gene expression data using real-time quantitative PCR and the 2(-Delta Delta C(T)) method. Methods 25: 402-408, 2001.

12. Kerr TA, Korenblat KM and Davidson NO: MicroRNAs and liver disease. Transl Res 157: 241-252, 2011.

13. Huang $\mathrm{S}$ and $\mathrm{He} \mathrm{X}$ : The role of microRNAs in liver cancer progression. Br J Cancer 104: 235-240, 2011.

14. Di Leva G and Croce CM: miRNA profiling of cancer. Curr Opin Genet Dev 23: 3-11, 2013.

15. Shih TC, Tien YJ, Wen CJ, Yeh TS, Yu MC, Huang $\mathrm{CH}$, Lee YS, Yen TC and Hsieh SY: MicroRNA-214 downregulation contributes to tumor angiogenesis by inducing secretion of the hepatoma-derived growth factor in human hepatoma. J Hepatol 57: 584-591, 2012.
16. Zeng XC, Liu FQ, Yan R, Yi HM, Zhang T, Wang GY, Li Y and Jiang N: Downregulation of miR-610 promotes proliferation and tumorigenicity and activates $\mathrm{Wnt} / \beta$-catenin signaling in human hepatocellular carcinoma. Mol Cancer 13: 261, 2014.

17. Reya $\mathrm{T}$ and Clevers H: Wnt signalling in stem cells and cancer. Nature 434: 843-850, 2005.

18. Hashemi Gheinani A, Burkhard FC, Rehrauer H, Aquino Fournier C and Monastyrskaya K: MicroRNA miR-199a-5p regulates smooth muscle cell proliferation and morphology by targeting Wnt2 signaling pathway. J Biol Chem 13: 7067-7086, 2015.

19. Qu B, Liu BR, Du YJ, Chen J, Cheng YQ, Xu W and Wang XH: Wnt/ $\beta$-catenin signaling pathway may regulate the expression of angiogenic growth factors in hepatocellular carcinoma. Oncol Lett 7: 1175-1178, 2014.

20. de La Coste A, Romagnolo B, Billuart P, Renard CA, Buendia MA, Soubrane O, Fabre M, Chelly J, Beldjord C, Kahn A and Perret C: Somatic mutations of the beta-catenin gene are frequent in mouse and human hepatocellular carcinomas. Proc Natl Acad Sci USA 95: 8847-8851, 1998.

21. Miyoshi Y, Iwao K, Nagasawa Y, Aihara T, Sasaki Y, Imaoka S, Murata M, Shimano T and Nakamura Y: Activation of the beta-catenin gene in primary hepatocellular carcinomas by somatic alterations involving exon 3. Cancer Res 58: 2524-2527, 1998.

22. Shi X and Garry DJ: Muscle stem cells in development, regeneration, and disease. Genes Dev 20: 1692-1708, 2006.

23. Tanaka S, Terada K and Nohno T: Canonical Wnt signaling is involved in switching from cell proliferation to myogenic differentiation of mouse myoblast cells. J Mol Signal 6: 12, 2011.

24. Anastas JN and Moon RT: WNT signalling pathways as therapeutic targets in cancer. Nat Rev Cancer 13: 11-26, 2013.

25. Li D, Liu W, Wang X, Wu J, Quan W, Yao Y, Bals R, Ji S, Wu K, Guo J and Wan H: Cathelicidin, an antimicrobial peptide produced by macrophages, promotes colon cancer by activating the Wnt/ $\beta$-catenin pathway. Oncotarget 6: 2939-2950, 2015.

26. Gougelet A and Colnot S: A complex interplay between Wnt $/ \beta$-catenin signalling and the cell cycle in the adult liver. Int J Hepatol 2012: 816125, 2012.

27. He TC, Sparks AB, Rago C, Hermeking H, Zawel L, da Costa LT, Morin PJ, Vogelstein B and Kinzler KW: Identification of c-MYC as a target of the APC pathway. Science 281: 1509-1512, 1998.

28. Constantinou T, Baumann F, Lacher MD, Saurer S, Friis R and Dharmarajan A: SFRP-4 abrogates Wnt-3a-induced beta-catenin and Akt/PKB signalling and reverses a Wnt-3a-imposed inhibition of in vitro mammary differentiation. J Mol Signal 3: 10, 2008.

29. Pan LH, Yao M, Cai Y, Gu JJ, Yang XL, Wang L and Yao DF: Oncogenic Wnt3a expression as an estimable prognostic marker for hepatocellular carcinoma. World J Gastroenterol 22: 3829-3836, 2016. 